\title{
Hemispheric differences in the interference among components of compound gratings
}

\author{
FREDERICK L. KITTERLE \\ Stephen F. Austin State University, Nacogdoches, Texas \\ and \\ STEPHEN CHRISTMAN and JORGE CONESA \\ University of Toledo, Toledo, Ohio
}

\begin{abstract}
The relationship between local/global and high/low spatial-frequency processing in hemispheric asymmetries was explored. Subjects were required to judge the orientation of a high- or low-spatialfrequency component of a compound grating presented in the left visual field (LVF) or right visual field (RVF). In Experiment 1, attention was focused on one or the other component. A signal detection analysis indicated that sensitivity $\left(d^{\prime}\right)$ to the high-spatial-frequency target was reduced more by the presence of the low-spatial-frequency component when both were presented in the LVF rather than in the RVF. In Experiment 2, subjects determined whether a target orientation was present, independent of spatial frequency at only a single level (i.e., at the high-or low-spatialfrequency level), as opposed to both or neither level. An RVF/LH (left hemisphere) advantage was found when the decision was based on the orientation of the high-frequency component. The asymmetrical influence of visual field of presentation and spatial frequency upon sensitivity is discussed in terms of hemispheric differences in the magnitude of inhibition between spatialfrequency channels and in the role of transient channel activity to capture and direct higher order attentional processes.
\end{abstract}

Hierarchical stimuli, in which a large character or stimulus is constructed in outline form from smaller characters or stimuli, have been used in perceptual studies to determine the nature of information-processing priorities (Navon, 1977; see also Shepp \& Ballesteros, 1989). For example, a large letter $\mathrm{H}$ may be constructed from smaller $\mathrm{Hs}$ or smaller Ss. The information contained in such a stimulus is at different scales-a large (global) scale in the case of the large $\mathrm{H}$ and a small (local) scale in the case of the small Hs or Ss. Information at the global and local levels is distinct yet embedded within each other.

The nature of the task in a given experiment may emphasize different aspects of a hierarchical stimulus. The subject's response may be based upon the information at the global level, the local level, or both. The responses to global and local stimuli could be compatible, incompatible, or neutral with respect to each other (Navon, 1977). Of theoretical interest in such an experiment is which component of information, local or global, has a processing advantage.

Studies have shown that there is a "global precedence" effect, in which information at the global level is processed

This research was partially supported by an Academic Challenge Award to the University of Toledo Department of Psychology to enhance research in experimental psychology, and by a subcontract to F.L.K. from a State of Ohio Department of Aging grant to the Medical College of Ohio. J. C. was supported by a Patricia Roberts Harris Fellowship. Address correspondence to F. L. Kitterle, Department of Psychology, Stephen F. Austin State University, Nacogdoches, TX 75962. faster than that at the local level. There can also be interference in the processing of local information by irrelevant information at the global level (i.e., "global interference"'; Miller, 1981; Navon, 1977; Robertson \& Lamb, 1991). A number of studies have delineated the conditions under which global precedence may occur as well as its possible mechanisms (Lamb \& Robertson, 1989, 1990; see Robertson \& Lamb, 1991, for a review; see Shepp \& Ballesteros, 1989, for a selection of papers).

It has been suggested that part of the explanation of this effect is perceptual and may reflect the properties of spatial-frequency channels in the human visual system (Hughes, 1986; Hughes, Fendrich, \& Reuter-Lorenz, 1990; Hughes, Layton, Baird, \& Lester, 1984; Robertson \& Lamb, 1991; Sergent, 1982). This idea derives from psychophysical and electrophysiological studies that have shown that the human visual system contains stimulusanalyzing mechanisms that respond to the spatial-frequency content in the visual stimulus (Blakemore \& Campbell, 1969; Campbell \& Robson, 1968; Kulikowski \& Tolhurst, 1973; Lehmkuhle, Kratz, Mangel, \& Sherman, 1980; Lennie, 1980; Singer \& Bedworth, 1973; Tolhurst, 1973). In terms of the processing of hierarchical stimuli, channels with low spatial frequency have been hypothesized to be the mechanisms that respond to large, global information whereas those with high spatial frequency are thought to analyze small, local detail. Thus, the output of these multiple spatial-frequency channels provides the brain with information represented at a number of scales and the nature of the task determines the level of infor- 
mation (e.g., local or global) or range of spatial frequencies (high or low) necessary for effective performance.

The temporal properties of low-spatial-frequency channels and/or asymmetrical inhibition between channels (e.g., low-spatial-frequency channels inhibit high-spatialfrequency channels) may account for the global precedence effect. It might be argued that global precedence arises as a result of the fact that low-spatial-frequency channels have a shorter latency and shorter temporal integration time than channels responding to high spatial frequencies (Breitmeyer, 1975). Responses to global information would be faster than those to local information. In addition, psychophysical and electrophysiological studies have demonstrated that low-spatial-frequency channels can inhibit activity in high-spatial-frequency channels (e.g., Breitmeyer, 1984; Hughes, 1986). Thus, even if the latencies of the local and global channels were equal, a global advantage still might occur as a result of global interference in the processing of local information.

Recent research has supported an explicit link between the processing of spatial frequency, on the one hand, and local/global information, on the other. In one study, Shulman, Sullivan, Gish, and Sakoda (1986) found that adaptation to a low-spatial-frequency grating interfered with the processing of global but not local information in a hierarchical display composed of large letters constructed from smaller letters. Adaptation to a high-spatial-frequency grating, on the other hand, impaired processing of information at the local but not the global level. In another study (Shulman \& Wilson, 1987), the primary task required subjects to detect a target letter at either the local or global level. A probe stimulus-a low-contrast highor low-spatial-frequency grating-immediately followed the hierarchical stimulus. The secondary task was to detect the probe. Shulman and Wilson (1987) found that the detection of a low-spatial-frequency grating was enhanced when the task focused attention on the processing of global information, whereas the detection of the high-spatialfrequency grating was enhanced when attention was focused on the processing of local stimulus information. Hughes et al. (1990) designed hierarchical stimuli with and without low-spatial-frequency information. The former condition yielded global precedence, whereas local precedence was found with the latter. Thus, when low-spatialfrequency information is removed, or low-spatial-frequency channels are adapted, a global advantage in processing is not found. These results suggest that the processing of local versus global information is at least partially dependent on the processing of high versus low spatial frequencies, respectively.

Neuropsychological research with brain-damaged populations and laterality research on normal populations has addressed the issue of local/global processing with the conclusion that the two hemispheres of the brain may differ in the efficiency with which they process local and global stimuli (Lamb \& Robertson, 1989; Lamb, Robertson, \& Knight, 1989, 1990; Robertson \& Delis, 1986; Robertson, Lamb, \& Knight, 1988). The left hemisphere
(LH) is more efficient at processing local detail, whereas the right hemisphere $(\mathrm{RH})$ has been found to be more efficient at processing information at the global level (Martin, 1979; Sergent, 1982; Van Kleeck, 1989). A common framework for synthesizing the links between local/global processing and high/low spatial-frequency channels, on the one hand, and between local/global processing and $\mathrm{LH} / \mathrm{RH}$ processes, on the other, is suggested by the finding that the left hemisphere is more efficient at processing high spatial frequencies and the right hemisphere is more efficient at processing low spatial frequencies (Kitterle \& Christman, 1991; Kitterle, Christman, \& Hellige, 1990; Sergent, 1982). These data reinforce the hypothesis that hemispheric differences in the processing of spatial-frequency information may underlie hemispheric differences in the processing of local and global stimuli (Robertson \& Lamb, 1991; Sergent, 1982, 1985).

The two experiments in the present study were designed to explore further the relationship between local/global and high/low spatial-frequency processing by the cerebral hemispheres using compound grating patterns and a task similar to that employed by Hughes (1986). Hughes measured reaction time to judge the orientation (horizontal vs. vertical) of a low- or high- (.5 and 5 cycles per degree [cpd]) spatial-frequency component of a foveally presented, compound grating. The other component of the stimulus could be in either the same orientation as the target component or orthogonal to it. He found asymmetric interference between components of suprathreshold gratings. The reaction times to discriminate orientation were significantly longer when the target was a high-spatialfrequency component and the irrelevant stimulus was an orthogonal, low-spatial-frequency grating, whereas the time to discriminate the orientation of the low-spatialfrequency grating was not influenced by an orthogonal high-spatial-frequency component. This finding mirrors the asymmetrical interference effects found in local/global experiments employing hierarchical stimuli.

On the basis of the above considerations and our earlier work on hemispheric differences in the processing of spatial frequency, the present experiments were designed to determine the influence of visual field of presentation upon the magnitude and direction of the aforementioned asymmetrical interference effect. In the present study, we measured the magnitude of the interference effect using accuracy and reaction time measures. A signal detection analysis was conducted on the accuracy data, motivated by two considerations. One consideration has to do with the nature of the accuracy measure. In particular, measures of accuracy are influenced by both the sensitivity of the observer $\left(d^{\prime}\right)$ and the decision criteria (beta) used by the observer in making a response. A signal detection analysis permits evaluation of the independent contribution of each of these components. A second consideration is based upon recent research that indicated that within a signal detection framework, hemispheric differences in response bias exist independently of hemispheric differences in sensitivity (Chiarello, Senehi, \& Soulier, 1986; 
Peterzell, Harvey, \& Hardyck, 1989). For example, Peterzell et al. found hemispheric asymmetries in response bias to bandpass filtered letters, but hemispheric symmetry in sensitivity. Thus, it is of theoretical importance to determine whether hemispheric differences in the interaction between spatial-frequency channels reflect differences in response bias, sensitivity, or both.

\section{EXPERIMENT 1 \\ Focused Attention}

In Experiment 1, we extended the paradigm of Hughes (1986) to address the question of whether there are hemispheric differences in the magnitude and direction of interference between the low- and high-spatial-frequency components of a compound grating. As in Hughes, the present experiment focuses attention on the output of a given spatial-frequency channel because it involves the judgment of the orientation of a specific component of the compound pattern. Both components of the pattern could be in the same orientation (congruent condition) or in orthogonal orientations (noncongruent condition). The compound pattern could be presented in the left visual field (LVF) or the right visual field (RVF). This generates a 2 (spatial frequency: judge orientation of high or low spatial frequency) $\times 2$ (condition: congruent vs. noncongruent) $\times 2$ (visual field: left vs. right) design.

On the basis of previous research (Hughes, 1986; Hughes et al., 1990), there should be asymmetrical interference, with the low-spatial-frequency component interfering more with the processing of the high-spatialfrequency component than the reverse. (In the present study, as in Hughes, 1986, interference is based upon the difference in performance between the noncongruent and congruent conditions.) Moreover, the research of Kitterle et al. (1990) suggests that low spatial frequencies should interfere more with the processing of high spatial frequencies when the compound pattern is presented in the LVF/RH rather than in the RVF/LH. In contrast, the processing of low-spatial-frequency orientation should show negligible interference from the high-frequency component for LVF presentations, whereas there might be a small influence of the high-spatial-frequency component with RVF presentations. Thus, the interference effect should depend upon both spatial frequency and visual field, suggesting a significant interaction between task, congruent/noncongruent conditions, and visual field.

\section{Method}

Apparatus and visual display. The spatial-frequency components of the stimuli were sinusoidal. The gratings were generated by a Picasso CRT image synthesizer (Innisfree) under computer control and were displayed on Tektronix 608 monitors. A refresh rate of $200 \mathrm{~Hz}$ was employed. A large black matte surround $\left(30^{\circ} \times\right.$ $36^{\circ}$ at a viewing distance of 42 in.) was placed directly in front of the monitors. Holes in the surround were used to mask the monitor screens down to two $6.8^{\circ}$ circular apertures. A small red LED placed between the two monitors served as the fixation point, and the inner edge of each monitor screen was $2.0^{\circ}$ from fixation.

The set of stimuli consisted of four compound gratings yielded by the factorial combination of two levels of spatial frequency (1.5 and
$9.0 \mathrm{cpd})$ and two levels of orientation $\left(45^{\circ}\right.$ and $\left.135^{\circ}\right)$. Oblique orientations were chosen for use in the present study to avoid the presence of luminance cues at the inner edges of the displays (with vertical/ horizontal orientations, horizontal gratings have a luminance gradient at the inner edge nearest the fovea, whereas vertical gratings have a constant luminance at the edge of the display).

The mean luminance and contrasts of the displays were calibrated by means of a Tektronix J16 digital photometer and a J6523 narrowangle luminance probe. The mean luminance of the displays was $10.3 \mathrm{~cd} / \mathrm{m}^{2}$. The contrast $(C)$ of the low-spatial-frequency component was 0.1 and that of the high-spatial-frequency component was 0.5 (where $C=$ [maximum luminance - minimum luminance]/ [maximum luminance + minimum luminance]). These contrasts were chosen because previous research indicates that they should yield equivalent reaction times. Care was taken during the course of the experiment to ensure that both CRTs were matched in luminance and contrast, and that the mean luminance of the display did not change with grating presentation. Stimuli were exposed for $160 \mathrm{msec}$ and were presented at a variable foreperiod $(600-900 \mathrm{msec})$ following a brief warning tone.

Procedure. The subjects viewed the displays through a viewing hood with a padded chinrest under near-dark conditions. Viewing was binocular. The importance of maintaining fixation on the fixation point was stressed to the subjects, who were told that the purpose of the experiment was to see how well people could identify stimuli without looking directly at them. At the beginning of the experiment, the subjects were light-adapted for $2 \mathrm{~min}$ to the mean luminance of the display.

The experiment consisted of low- and high-frequency conditions. In the low-frequency condition, the subjects responded to the orientation of the low-frequency component of the input, depressing one response key if it was at $45^{\circ}$ and the other if it was at $135^{\circ}$. In the high-frequency condition, they responded similarly to the orientation of the high-frequency component. The order of conditions and hand of response were counterbalanced across subjects. All the subjects used the same orientation assignment (e.g., left key for $45^{\circ}$ and right key for $135^{\circ}$ ) for both conditions to minimize negative transfer across conditions. They were instructed to respond as quickly and as accurately as possible. Both percent correct and reaction time (RT) measures were collected.

Each condition consisted of a block of 160 trials, resulting from the factorial combination of four different stimuli, two visual fields, five foreperiods, and four replications. The subjects were also given practice blocks of 24 trials before each condition to help familiarize them with the nature of the task and stimuli.

Subjects. Four male and 4 female subjects with normal or corrected-to-normal vision served as observers. All were righthanded with no left-handed relatives in their immediate family, as assessed by a brief handedness questionnaire. The subjects were drawn from the University of Toledo psychology department's human subject pool, were naive about the purpose of the experiment, and received course credit for their participation.

\section{Results}

As Peterzell et al. (1989) have cautioned, interpretations of hemispheric differences in processing spatialfrequency information based upon an accuracy indicator such as percent correct are at best ambiguous. These asymmetries can be attributed to sensitivity differences between the hemispheres, different spatial frequencies, or to differences in response bias. This ambiguity suggests that a signal detection analysis of performance is needed in order to determine whether hemispheric asymmetries reflect differences in sensitivity, differences in response bias, or both. Consequently, we conducted a signal detection analysis of the data and present the results 
below. These analyses are based upon 20 replications of each of the eight conditions ( 2 target spatial frequencies $\times 2$ consistent/inconsistent orientations of the irrelevant spatial frequency component $\times 2$ visual fields). Analyses of the reaction time data are also presented.

The rationale used in conducting the signal detection analysis for this experiment is as follows. The task of the subjects was to judge whether a given spatial frequency was presented at a $45^{\circ}$ or $135^{\circ}$ orientation. Thus, if the sensory magnitude elicited by a grating presented at $45^{\circ}$ is higher than at $135^{\circ}$, it is more likely that the grating was presented in the former orientation than in the later. However, if the sensory magnitude is greater for the $135^{\circ}$ orientation, it is more likely that the grating was presented in this orientation rather than at $45^{\circ}$. Thus, it is sufficient to specify the information available to the sensory system in terms of the magnitude of the response to a grating oriented at $45^{\circ}$. In this way, we define a "hit" as a response that a given grating is presented at $45^{\circ}$ when it is presented at that orientation, and define a "false alarm" as a response that the grating is at $45^{\circ}$ when it is actually presented at $135^{\circ}$. From these data, then, we have derived measures of $d^{\prime}$ and beta.

Table 1 summarizes the results of this experiment for $d^{\prime}$ and beta for each condition of Experiment 1. A threefactor repeated measures analysis of variance (ANOVA) with a 2 (target spatial frequency) $\times 2$ (consistency) $\times$ 2 (visual field) design was performed on the data.

Analysis of $d^{\prime}$. The main effect of spatial frequency was significant $[F(1,7)=17.66, p<.009]$. This indicates that sensitivity to the high-spatial-frequency target was less than sensitivity to the low-spatial-frequency grating.

The significant second-order interaction between spatial frequency, consistency, and visual field indicates that the sensitivity to a given target's spatial frequency was also influenced by the orientation of the nontarget component and by the visual field in which the compound grating was presented $[F(1,7)=7.56, p<.04]$. A fine-grain analysis revealed the source of this interaction. For the 1-cpd target, there was an increase in sensitivity (4.946.00) when the orientation of the target and the nontarget components were changed from a consistent to inconsistent orientation $[F(1,7)=9.27, p<.01]$ with LVF presentations. Conversely, there was a small decrease in sensitivity (5.48 vs. 4.69 ) for a shift in orientation from consistent to inconsistent for RVF presentations $[F(1,7)=$ $1.83, p>.06]$. With the 9-cpd target, the change in orien-

Table 1

Mean Reaction Time (in Milliseconds) as a Function of Stimulus Condition for 1- and 9-cpd Targets

\begin{tabular}{cccccc}
\hline & \multicolumn{2}{c}{ Target Spatial Frequency } \\
\cline { 2 - 3 } \cline { 5 - 6 } Visual Field & \multicolumn{2}{c}{1 cpd } & & \multicolumn{2}{c}{$9 \mathrm{cpd}$} \\
\cline { 2 - 3 } \cline { 5 - 6 } LVF & Consistent & Inconsistent & & Consistent & Inconsistent \\
\hline RVF & 402 & 483 & & 578 & 695 \\
\hline
\end{tabular}

Note - LVF $=$ left visual field; RVF $=$ right visual field.
Table 2

Signal Detection Analysis for Experiment 1 Showing Beta and $d^{\prime}$ as a Function of Stimulus Condition

\begin{tabular}{|c|c|c|c|c|}
\hline \multirow[b]{3}{*}{ Visual Field } & \multicolumn{4}{|c|}{ Target Spatial Frequency } \\
\hline & \multicolumn{2}{|c|}{$1 \mathrm{cpd}$} & \multicolumn{2}{|c|}{$9 \mathrm{cpd}$} \\
\hline & Consistent & Inconsistent & Consistent & Inconsistent \\
\hline \multicolumn{5}{|c|}{ Beta } \\
\hline LVF & 1.61 & 1.00 & .42 & .71 \\
\hline RVF & 1.68 & 1.82 & .94 & .71 \\
\hline \multicolumn{5}{|c|}{$d^{\prime}$} \\
\hline LVF & 4.94 & 6.00 & 3.68 & 2.38 \\
\hline RVF & 5.48 & 4.69 & 3.26 & 3.18 \\
\hline
\end{tabular}

Note-LVF = left visual field; RVF $=$ right visual field.

tation from consistent to inconsistent produced a significantly marked decrease in sensitivity $(3.68-2.38)[F(1,7)$ $=15.95, p<.01]$ for LVF but not RVF presentations. With RVF presentations, there were no significant changes in sensitivity.

Analysis of beta. A three-factor ANOVA was carried out for log-transformed beta measures, presented in Table 1 . The data were transformed because of the extremely skewed nature of this measure (Macmillan \& Creelman, 1991). The results of this analysis indicate that there was a significant effect of spatial frequency $[F(1,7)=5.11$, $p<.05]$. No other effects were found to be significant. Unlike Peterzell et al. (1989), we did not find any differences in visual field for this measure.

Reaction time data. The mean reaction times for all conditions are summarized in Table 2 . Because the mean RTs were linearly related to the standard deviation, they were logarithmically transformed and then subjected to a three-factor repeated measures ANOVA. There was a highly significant spatial frequency $\times$ condition $\times$ visual field interaction $[F(1,7)=6.83, p<.03]$. The results shown in Figure 1 plot the interference effect (the difference in RT between the incongruent and congruent conditions) as a function of the spatial frequency of the target for LVF presentations (filled circles) and RVF presentations (open circles).

Note that there are hemispheric processing asymmetries with this target. There was virtually no interference in the processing of the low-spatial-frequency target for LVF/ RH presentations $(F<1)$. However, RVF/LH presentations resulted in significant interference in the processing of the low-spatial-frequency target by the nontarget highspatial-frequency component $[F(1,7)=11.06, p<.01]$.

The asymmetrical nature of interference is also evident in this figure. A more dramatic interference effect was found when the target was high spatial frequency and the nontarget component was low spatial frequency rather than the reverse. It is also evident that there are hemispheric asymmetries in the magnitude of the interference effect. It was greater when the high-spatial-frequency target was presented in the LVF/RH $[F(1,7)=16.21, p<$ $.005]$, but also occurred for RVF/LH presentations $[F(1,7)$ $=6.39, p<.03]$. 


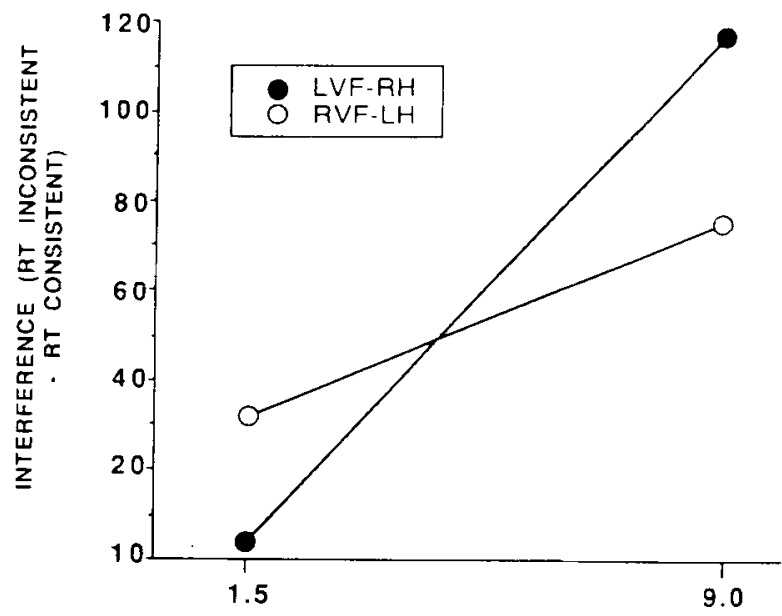

TARGET SPATIAL FREQUENCY (c/deg)

Figure 1. Reaction time results for Experiment 1. Interference effect (RT incongruent condition-RT congruent condition) as a function of target spatial frequency for LVF-RH (filled circles) and RVFLH (open circles).

In summary, the results of a signal detection analysis indicate that processing of the orientation of a target spatial frequency in a compound grating is influenced by the presence of the other spatial-frequency component and by the visual field in which the pattern is presented. These effects are revealed as changes in sensitivity and reaction time. Specifically, there was greater interference from low-spatial-frequency components and visual field on the processing of high-spatial-frequency targets than the reverse, and the processing of high-frequency targets was disrupted more with LVF than with RVF presentations. On the other hand, high-spatial-frequency components interfered with the processing of the low-spatial-frequency target only for RVF presentations. However, on LVF presentations, the processing of low-spatial-frequency orientation was not influenced by the high-spatial-frequency component.

\section{EXPERIMENT 2}

\section{Divided Attention}

In Experiment 1, the orientation of a specific spatial frequency was determined, which mirrors studies that have used hierarchical stimuli composed of letters in which subjects are required to identify the letter at a particular level (i.e., local or global). Because the task requires the identification of the orientation of a designated target spatial frequency, we have referred to this as a focused-attention task. In contrast, other studies that have used hierarchical stimuli composed of letters have designated a specific letter as a target and required subjects to indicate whether the letter was presented on a given trial, regardless of whether it was at the local or global level. This class of experiments has been referred to as divided-attention tasks because the target can appear at the local or global level, both levels, or neither level. Thus, Experiment 2 was designed as a divided-attention task involving the determination of whether a target orientation was present, independent of spatial frequency. There were two target $\left(45^{\circ}\right.$ and $\left.135^{\circ}\right)$ and two nontarget $\left(0^{\circ}\right.$ and $\left.90^{\circ}\right)$ orientations. There were 16 possible patterns formed by combining a low-spatial-frequency grating at $45^{\circ}, 135^{\circ}, 0^{\circ}$, or $90^{\circ}$ with a high-spatial-frequency grating at $45^{\circ}, 135^{\circ}, 0^{\circ}$, or $90^{\circ}$. For a given compound grating, both, one, or neither of the spatial-frequency components could be at the target orientation. Thus, target orientation could be at the local level, the global level, both levels, or neither level.

This experiment was designed specifically to mirror the procedure employed by Sergent (1982). In her experiment, the stimulus set consisted of four letters, two of which were designated as targets. Target letters could appear at the local, global, both, or neither level. The subject's task was to detect the presence of the target orientation at any level. In the present experiment, four orientations were substituted for the four letters and two of them were designated as target orientations. The subject's task was to detect the presence of a target orientation at the highand/or low-spatial-frequency component.

In this experiment, the crucial comparisons involved the conditions in which the target orientation was present at only a single level (i.e., at the local or global level), as opposed to both or neither level. It was hypothesized that an LH advantage should emerge when the decision was based on the orientation of the high-frequency component alone, and an RH advantage should be obtained for decisions based on the low-spatial-frequency component alone.

\section{Method}

Apparatus and visual display. The apparatus and general viewing conditions were the same as those described in Experiment 1, with the following changes. The stimuli consisted of compound gratings containing a low-frequency $(1.5 \mathrm{cpd})$ and a high-frequency (9.0 cpd) sinusoidal component; the total stimulus set consisted of a total of 16 different stimuli, resulting from the factorial combination of four possible orientations of the low-frequency component $\left(0^{\circ}, 45^{\circ}, 90^{\circ}\right.$, and $\left.135^{\circ}\right)$, and four possible orientations of the high-frequency component $\left(0^{\circ}, 45^{\circ}, 90^{\circ}\right.$, and $\left.135^{\circ}\right)$. The stimuli were exposed for $180 \mathrm{msec}$ and were presented after a variable foreperiod $(600-1,000 \mathrm{msec})$ following a brief warning tone.

Procedure. Orientations of $45^{\circ}$ and $135^{\circ}$ were designated as targets. The subjects were instructed to press a key with one hand if a target orientation was present at the low-and/or high-frequency level; if neither of the components of the stimulus had target orientations (i.e., were both $0^{\circ}$ and/or $90^{\circ}$ ), they were to press another key with the other hand. For purposes of data analysis, the 16 factorial combinations of spatial frequency and orientation can be further reduced to conditions in which the target orientation appeared at both the low- and high-frequency levels $(\mathrm{L}+\mathrm{H}+)$, at only the low-frequency level $(\mathrm{L}+\mathrm{H}-)$, at only the high-frequency level $(\mathrm{L}-\mathrm{H}+)$, or at neither level $(\mathrm{L}-\mathrm{H}-)$. In addition, when target orientations occurred at both or neither level, the orientations of the low- and high-frequency components could be identical (Id.) 
or nonidentical (N.Id.). Thus, there were a total of six possible stimulus conditions: $\mathrm{L}+\mathrm{H}+$ (Id.), $\mathrm{L}+\mathrm{H}+(\mathrm{N} . \mathrm{Id}$.), $\mathrm{L}+\mathrm{H}-, \mathrm{L}-\mathrm{H}+$, $\mathrm{L}-\mathrm{H}-$ (Id.), and $\mathrm{L}-\mathrm{H}-$ (N.Id.). The stimulus set was constructed so that there was an equal number of target and nontarget stimuli. Hand of response was counterbalanced across subjects.

The experiment consisted of two blocks of 144 trials each, resulting from the factorial combination of 24 different stimuli (12 target stimuli, plus three replications each of 4 nontarget stimuli), two visual fields, and three foreperiods $(600,800$, and 1,000 msec). The foreperiods served as a replication factor, and were not entered into any subsequent analyses. The subjects were given a practice block of 24 trials before each experimental block to help familiarize them with the nature of the task and stimuli.

Subjects. Four male and 4 female subjects with normal or corrected-to-normal vision served as observers. All were righthanded with no left-handed relatives in their immediate family, as assessed by a brief handedness questionnaire. The subjects were drawn from the University of Toledo psychology department's human subject pool, were naive about the purpose of the experiment, and received course credit for their participation.

\section{Results}

As in Experiment 1, a signal detection analysis was applied to the accuracy data. Measures of $d^{\prime}$ and beta as well as reaction times were subjected to a 6 (pattern: $\mathrm{L}+\mathrm{H}+$ congruent, $\mathrm{L}+\mathrm{H}+$ incongruent, $\mathrm{L}+\mathrm{H}-, \mathrm{L}-\mathrm{H}+$, $\mathrm{L}-\mathrm{H}-$ congruent, and $\mathrm{L}-\mathrm{H}-$ incongruent) $\times 2$ (visual field) repeated measures analysis. Recall that $L$ and $H$ indicate low- and high-spatial-frequency components of the pattern, respectively. $\mathrm{L}+\mathrm{H}+$ signifies that both the lowand high-spatial-frequency components were targets, $\mathrm{L}+\mathrm{H}-$ indicates that the low-spatial-frequency component was a target and the high was not, $\mathrm{L}-\mathrm{H}+$ indicates that the low-frequency component was a nontarget and the high-frequency component was a target, and $\mathrm{L}-\mathrm{H}-$ indicates that both components were nontargets. Congruent and noncongruent refer to the compound gratings in which the components were in the same or orthogonal orientations, respectively.

The rationale used in conducting the signal detection analysis for this experiment was similar to the rationale employed in Experiment 1. We define a hit as a response that a given grating was presented in the target orientation when it was presented at that orientation, and define a false alarm as a response that the grating was at the target orientation when it was actually presented in a nontarget orientation. From these data, we have derived measures of $d^{\prime}$ and beta, which are presented in Table 3, and present the results of this analysis for all of the target conditions (i.e., $\mathrm{L}+\mathrm{H}+$ congruent, $\mathrm{L}+\mathrm{H}+$ incongruent, $\mathrm{L}+\mathrm{H}-, \mathrm{L}-\mathrm{H}+)$.

Analysis of $d^{\prime}$. A 4 (pattern) $\times 2$ (visual field) ANOVA on the $d^{\prime}$ measure indicated a significant main effect of pattern type $[F(3,21)=45.04, p<.0001]$. Sensitivity was greater for compound patterns when the target was of low spatial frequency, regardless of the nature of the high-spatial-frequency component, rather than for a pattern in which only the high-spatial-frequency component was the target. Thus, there is evidence in these data, as in the previous experiment, of an asymmetrical interaction between spatial-frequency channels: Low-spatial-frequency
Table 3

Signal Detection Analysis for Experiment 2 Showing Beta and $d^{\prime}$ as a Function of Stimulus Condition

\begin{tabular}{cccccc}
\hline & \multicolumn{5}{c}{ Patterns } \\
\cline { 2 - 6 } Visual Field & $\mathrm{L}+\mathrm{H}+($ Id.) & $\mathrm{L}+\mathrm{H}+(\mathrm{N} . \mathrm{Id})$. & $\mathrm{L}+\mathrm{H}-\mathrm{L}-\mathrm{H}+$ \\
\hline & \multicolumn{5}{c}{ Beta } \\
LVF & .14 & .27 & .2 & 3.8 \\
RVF & .13 & .13 & .24 & .95 \\
& & $d^{\prime}$ & & & \\
LVF & 4.08 & 3.88 & 3.72 & 1.16 \\
RVF & 3.88 & 3.77 & 3.65 & 1.72 \\
\hline
\end{tabular}

Note-LVF = left visual field; $\mathrm{RVF}=$ right visual field. $\mathrm{L}+\mathrm{H}+=$ target orientation at both high- and low-frequency levels; $\mathrm{L}+\mathrm{H}-=$ target orientation at only the low-frequency level; $\mathrm{L}-\mathrm{H}+=$ target orientation at only the high-frequency level. Id. = Identical; N.Id. = nonidentical.

components influence the processing of high-spatialfrequency targets, but not the reverse. The main effect of visual field was not significantly different $[F(1,7)=$ $2.77, p>.14]$. However, there was a significant pattern $\times$ visual field interaction $[F(3,21)=4.57, p<.01]$. This is due to the fact that there was a shift from an LVF sensitivity advantage for $\mathrm{L}+\mathrm{H}+, \mathrm{L}+\mathrm{H}-$ patterns to an $\mathrm{RVF}$ advantage for the $\mathrm{L}-\mathrm{H}+$ pattern.

It is also interesting to note that there was a consistent trend for all conditions in which the low-spatial-frequency grating was in the target orientation and that sensitivity was higher for LVF than for RVF presentations, regardless of whether the high-spatial-frequency component was or was not in a target orientation. On the other hand, only when the high-spatial-frequency grating was in the target orientation was there a shift in sensitivity from an LVF to an RVF advantage. Thus, sensitivity in the $\mathrm{L}+\mathrm{H}+$ and $\mathrm{L}+\mathrm{H}-$ conditions was primarily determined by the lowspatial-frequency component, although for both visual fields there is some indication that when the high-spatialfrequency component was in an orthogonal target orientation or nontarget orientation, sensitivity to the lowspatial-frequency component was slightly reduced.

Analysis of beta. Because of a correlation between the mean and standard deviation for the beta measure, an ANOVA on logarithmically transformed scores was carried out. The results of this analysis indicated the existence of a nonsignificant main effect of visual field $[F(1,7)=$ $5.11, p<.07]$. The effects of pattern type and the interaction between pattern type and visual field were not significant $(F<1)$.

As found in Experiment 1, interference effects were asymmetrical and depended upon visual field. The effects are revealed as changes in $d^{\prime}$ and thus indicate that the nature of the interactions between spatial-frequency channels and visual field are due to mechanisms that alter sensitivity to target presentation rather than visual-fielddependent criterion changes. Sensitivity to low-spatialfrequency gratings was greater for LVF presentations, whereas the high-spatial-frequency component appears to have had relatively little effect on processing. In contrast, 
sensitivity to high-spatial-frequency gratings was greatly reduced by the presence of the low-spatial-frequency component. Sensitivity reduction was greater for LVF than for RVF presentations. Thus, although the low-spatialfrequency component interfered with the processing of the high-spatial-frequency target, its effect was modulated by the visual field of presentation. Sensitivity was greater for RVF presentations of the high-spatial-frequency target.

In the present analysis, it should be noted that the number of errors were considerably higher than in Experiment 1 , thereby precluding a meaningful interpretation of the RT data for this experiment. The increase in RT and error rate suggests that the divided-attention task of Experiment 2 was a more demanding task than the focusedattention task of Experiment 1. Nevertheless, qualitatively similar interactions between spatial frequency and visual field were obtained in both experiments.

\section{DISCUSSION}

The results of the signal detection analyses for both experiments showed visual field $\times$ spatial-frequency interactions for $d^{\prime}$ but not beta, indicating that the hemispheric asymmetries found in both experiments cannot be attributed to differences between the hemispheres in decision criteria (beta) used by the observer in making a response. Rather, sensory interactions between spatial-frequency channels appears to alter sensitivity, as reflected in changes in $d^{\prime}$ and RT.

As noted earlier with central visual field presentations, Hughes (1986) found asymmetrical interference; that is, low-spatial-frequency channels interfered with the processing of high-spatial-frequency channels, but not the reverse. The present study has shown that the magnitude and direction of the interference between spatial-frequency channels depends upon the visual field of presentation. In Experiment 1, in which subjects judged the orientation of one of the two spatial-frequency components of a compound grating, two types of asymmetrical interference were found. The low-spatial-frequency component had a significantly greater interference effect on the processing of the high-spatial-frequency grating than the reverse. The strength of this interference was greater for LVF than for RVF presentations. On the other hand, although small in magnitude, the high-spatial-frequency component interfered with the processing of the lowspatial-frequency component. However, this effect was confined to RVF presentations. For LVF presentations, the high-spatial-frequency component had virtually no influence on the processing of the low-spatial-frequency target.

In Experiment 2, the subjects had to search for target orientations that could be present at either, neither, or both frequency levels. When the target orientation was present only at the high-frequency level, there was greater interference for LVF than for RVF presentations by the nontarget low-spatial-frequency component. In this task, the high-spatial-frequency component did not influence pro- cessing of the low-spatial-frequency component when it was presented in either the LVF or RVF.

It is quite possible that the asymmetrical interference effect found in these results can be completely explained in terms of response competition (Hughes, 1986). This would clearly be the case when the two gratings were in different orientations. Consequently, one would expect to find longer RTs to low-spatial-frequency targets in the inconsistent condition, in which response competition was maximal, compared with RTs to high-spatial-frequency targets in the consistent condition, in which response competition was minimal. However, as can be seen in Tables 1 and 2, the former condition yielded faster RTs than the latter, which holds for both visual fields. Moreover, the magnitude and direction of the interference depends not only upon spatial frequency, but also upon visual field. As Hughes (1986) has argued, if sensory effects accumulated at the same rate, and orthogonal gratings produced competing responses, we would expect competing response tendencies for both high- and low-spatial-frequency targets and, consequently, symmetrical interference effects. Moreover, competing response tendencies should not lead to hemispheric asymmetries. The presence of asymmetrical interactions between spatial-frequency channels that are dependent upon visual field of presentation does not imply that our results are not influenced by competing response tendencies. Rather, response competition has an effect that depends upon the rate at which earlier information about each component grating accumulates. Thus, the magnitude of response competition should depend upon hemispheric differences in sensitivity to spatial frequency. For example, there should be more response competition when the target grating is encoded by the less sensitive hemisphere, because of a difference in the rates at which information about the target and the nontarget grating accumulate. Note in Table 1 that RTs to high-spatialfrequency targets in the consistent condition presented in the RVF, in which response competition was minimal and sensitivity to the target's spatial frequency was maximal, were longer than those to low-spatial-frequency targets in the inconsistent condition presented in the RVF, in which response competition was maximal and sensitivity to the target was minimal.

Based upon the above considerations and the fact that contrast levels were chosen from previous research (see Kitterle et al., 1990) to equate the RTs to both spatialfrequency components presented in isolation, some aspect of the internal response to a given component is influenced by the presence of the other component and by the visual field of presentation. Hughes (1986) has argued that the interference effect may be attributed to inhibition of sustained channels by transient channels. Support for this idea is found in electrophysiological studies (Lehmkuhle et al., 1980; Lennie, 1980; Morrone, Burr, \& Maffei, 1982; Petrov, Pigarev, \& Zenkin, 1980; Singer $\&$ Bedworth, 1973). Moreover, the fact that interference occurs in a compound pattern when the component gratings are orthogonal may be related to "cross-orientation 
inhibition" in cortical cells (Morrone et al., 1982; Petrov et al., 1980), where it has been found that the neural responses to a grating in an optimal orientation can be reduced by band-limited noise or a second grating widely separated from the first in orientation or spatial frequency. In addition, psychophysical data support this conclusion by demonstrating that the interference effect is abolished when transient activity is reduced (Hughes, 1986; Paquet \& Merikle, 1984) or when low-spatial-frequency components are filtered from the stimulus display (Hughes et al., 1990). The fact that interference effects depend upon visual field suggests that the magnitude of interchannel inhibition differs for the two hemispheres.

Peterzell et al. (1989) have shown that visual field differences to spatial-frequency-filtered letter stimuli reflect asymmetries in response bias to spatial frequency rather than to sensitivity. These results raise important questions about the nature of the analysis used to evaluate and characterize the mechanisms involved in cerebral laterality effects (i.e., whether they operate in the perceptual or decision process). Despite their findings, the results of Peterzell et al. leave important questions unanswered. For example, studies of hemispheric differences to spatial frequency typically have shown an interaction between spatial frequency and visual field. However, Peterzell et al. found a main effect of visual field only for the measure of response bias-beta. Clearly, if response bias totally accounted for the interaction between visual field and spatial frequency, one would expect to see a visual field $x$ spatial frequency interaction for the dependent measurebeta. Thus, other non-response-bias mechanisms must determine the nature of the cerebral laterality effects to spatial frequency, namely, sensitivity differences. Our results indicate that the mechanism for attenuating sensitivity may be a hemispheric difference in modulating interchannel inhibition. It is evident from recent electrophysiological studies that the pattern of these interactions can be quite complex, depending upon the spatial frequencies of the gratings used, their orientation, and the cortical cells studied (Gilbert \& Wiesel, 1990).

One of the possible reasons for the discrepancy between the present study and that of Peterzell et al. (1989) is that the discrimination between target and nontarget letters could be made by detecting whether a local feature was present (i.e., a horizontal line at the top of the letter). If subjects could perform this task on the basis of simply detecting whether or not that bar was present, then hemispheric differences would not be expected because detection, not identification, is involved and our earlier work has shown that hemispheric symmetry is obtained in detection tasks (Kitterle et al., 1990). We are currently testing that idea.

The results of the signal detection analysis are consistent with our earlier interpretation that hemispheric processing asymmetries for spatial frequency may result, in part, from computational differences between the hemi- spheres in identifying and discriminating outputs of spatialfrequency channels (Kitterle et al., 1990).

However, although we have argued above that sensitivity to a given target can be altered by inhibition, there is another possible interpretation of these effects in terms of attentional mechanisms as opposed to interchannel inhibition. That is, the transient activity generated by lowspatial-frequency channels may serve to capture and direct higher order attentional processes independent of any spatial analysis of the stimulus per se (Breitmeyer, 1984; Jonides \& Yantis, 1988). Consequently, in the focusedattention task of Experiment 1, for example, this momentary shift of attention to the low-spatial-frequency channel could result in a longer reaction time to the highspatial-frequency target independent of any interchannel interaction. Thus, conditions that reduce the output of lowspatial-frequency channels (e.g., filtering of low spatial frequencies, flicker adaptation, ramping on stimuli) eliminate this attention-capturing transient activity and consequently reduce or abolish the interference effect. There is some support for the role of attentional processes in global precedence (Miller, 1981). Alternatively, it may well be the case that both processes operate; activity in low-spatial-frequency channels may direct attention and, thus, lead to global precedence, whereas the asymmetric interference of low-spatial-frequency channels on highspatial-frequency channels may reflect global interference and inhibit activity in high-spatial-frequency channels. This interpretation may account for the findings of Lamb et al. (1990) and Robertson and Lamb (1991) that global precedence and global interference are dissociable processes. Brain damage in one region of the brain affects global precedence and not global interference, whereas brain damage in another region of the brain abolishes global interference but not global precedence. In addition, Hughes et al. (1984) have shown that when the nontarget component of a hierarchical stimulus is held constant across trials, global precedence is reduced but not eliminated. It may be that under this condition, the inhibition between channels is still present but that the additional influence of the attention-directing transient activity can be ignored, resulting in a reduced global precedence effect.

In summary, the present experiments are consistent with the suggestion that hemispheric differences in local versus global processing are due partially to corresponding hemispheric differences in the processing of high versus low spatial frequency, respectively. Currently, we are extending this investigation to an explicit consideration of the role of the temporal characteristics of low-spatial-frequency channels. Previous work has shown that decreasing the transient response of the low-spatial-frequency channels decreases the magnitude of the asymmetrical interference of low spatial frequencies on the processing of high spatial frequencies (Hughes, 1986). An extension of this notion to examinations of hemispheric differences in local/global processing is an important next step, because research has 
shown that the hemispheres differ in their response characteristics to variations in temporal, as well as spatial, frequency (Rebai, Mecacci, Bagot, \& Bonnet, 1989).

\section{REFERENCES}

Blakemore, C., \& Campeell, F. W. (1969). On the existence of neurons in the human visual system selectively sensitive to the orientation and size of retinal images. Journal of Physiology, 203, 237-260.

Breitmeyer, B. G. (1975). Simple reaction time as a measure of the temporal response properties of transient and sustained channels. $\mathrm{Vi}$ sion Research, 15, 1411-1412.

BREITMEYER, B. G. (1984). Visual masking: An integrative approach. New York: Oxford University Press.

Camprell, F. W., Robson, J. C. (1968). Application of Fourier analysis to the visibility of gratings. Journal of Physiology, 197, 551-566.

Chiarello, C., Senehi, J., \& Soulier, M. (1986). Viewing conditions and hemispheric asymmetry for lexical decision. Neuropsychologia, 24, 521-530.

GilberT, C. D., \& Wiesel, T. N. (1990). The influence of contextual stimuli on the orientation selectivity of cells in the primary visual cortex of the cat. Vision Research, 30, 1689-1701.

Hughes, H. C. (1986). Asymmetric interference between components of suprathreshold compound gratings. Perception \& Psychophysics, 40, $241-250$.

Hughes, H. C., Fendrich, R., \& Reuter-Lorenz, P. A. (1990). Global versus local processing in the absence of low spatial frequencies. Journal of Cognitive Neuroscience, 2, 272-282.

Hughes, H. C., Layton, W. M., Baird, J. C., \& Lester, L. S. (1984). Global precedence in visual pattern recognition. Perception \& Psychophysics, 35, 361-371.

JoNidES, J., \& YANTIS, S. (1988). Uniqueness of abrupt visual onset in capturing attention. Perception \& Psychophysics, 43, 346-354.

Kitterie, F. L., \& Christman, S. (1991). Symmetries and asymmetries in the processing of sinusoidal gratings. In F. L. Kitterle (Ed.), Cerebral laterality: Theory and research, the Toledo symposium (pp. 201-224). Hillsdale, NJ: Erlbaum.

Kitterle, F. L., Christman, S., \&ellige, J. B. (1990). Hemispheric differences are found in the identification, but not the detection, of low versus high spatial frequencies. Perception \& Psychophysics, 48, 297-306.

KULIKowski, J. J., \& Tolhurst, D. J. (1973). Psychophysical evidence for sustained and transient detectors in human vision. Joumal of Physiology, 232, 149-162.

LAMB, M. R., \& Robertson, L. C. (1989). Do response time advantage and interference reflect order of processing of global- and locallevel information? Perception \& Psychophysics, 46, 254-258.

LAMB, M. R., \& RoBERTSON, L. C. (1990). The effect of visual angle on global and local reaction times depends on the set of visual angles presented. Perception \& Psychophysics, 47, 489-496

LAMB, M. R., RoBertson, L. C., \& KNIGHT, R. T. (1989). Attention and interference in the processing of global and local information: Effects of unilateral temporal-parietal junction lesions. Neuropsycholo gia, 27, 471-483.

Lamb, M. R., Robertson, L. C., \& KNIGHT, R. T. (1990). Component mechanisms underlying the processing of hierarchically organized patterns: Inferences from patient with unilateral cortical lesions. Journal of Experimental Psychology: Learning, Memory, \& Cognition, 16, 471-483.

Lehmkuhle, S., Kratz, K. E., Mangel, S. C., \& Sherman, S. M. (1980). Spatial and temporal sensitivity of $X$ and $Y$ cells in the dorsal lateral geniculate nucleus of the cat. Journal of Neurophysiology, 43, 520-541.
LENNIE, P. (1980). Parallel visual pathways: A review. Vision Research, 20, 561-594.

Macmillan, N. A., \& Creelman, C. D. (1991). Detection theory: A user's guide. New York: Cambridge University Press.

Martin, M. (1979). Hemispheric specialization for local and global processing. Neuropsychologia, 17, 33-40.

MILLER, J. (1981). Global precedence in attention and decision. Journal of Experimental Psychology: Human Perception \& Performance, 7, 1161-1174.

Morrone, M. C., Burr, D. C., \& Maffei, L. (1982). Function implications of cross-orientation inhibition of cortical visual cells: I. Neurophysiological evidence. Proceedings of the Royal Society of London: Series B, 216, 335-354.

Navon, D. (1977). Forest before trees: The precedence of global features in visual perception. Cognitive Psychology, 9, 353-383.

Paquet, L., \& Merikle, P. M. (1984). Global precedence: The effects of exposure duration. Canadian Joumal of Psychology, 38, 45-53.

Peterzell, D. H., Harvey, L. O., \& Hardyck, C. D. (1989). Spatial frequencies and the cerebral hemispheres: Contrast sensitivity, visible persistence, and letter classification. Perception \& Psychophysics, 46, 443-455.

Petrov, A. P., Pigarev, I. N., \& Zenkin, G. M. (1980). Some evidence against Fourier analysis as a function of the receptive fields in the cat's striate cortex. Vision Research, 20, 1023-1025.

Rebai, M., MeCaCCI, L., Bagot, J., \& BonNet, C. (1989). Influence of spatial frequency and handedness on hemispheric asymmetry in visually steady state evoked potentials. Neuropsychologia, 27, 315-334.

Robertson, L. C., \& Delis, D. C. (1986). "Part-whole" processing in unilateral brain-damaged patients: Dysfunction of hierarchical organization. Neuropsychologia, 24, 363-370.

RoBertson, L. C., \& LAMB, M. R. (1991). Neuropsychological perspectives on theories of part/whole organization. Cognitive Psychology, 23, 299-330.

Robertson, L. C., LAMB, M. R., \&Night, R. T. (1988). Effects of lesions of temporal-parietal junction on perceptual and attentional processing in humans. Journal of Neurosciences, 8, 3757-3769.

SERGENT, J. (1982). The cerebral balance of power: Confrontation or cooperation? Journal of Experimental Psychology: Human Perception \& Performance, 8, 253-272.

SERGENT, J. (1985). Influence of task and input factors on hemispheric involvement in face processing. Journal of Experimental Psychology: Human Perception \& Performance, 11, 846-861.

ShePP, B. E., BAllesteros, S. (Eds.) (1989). Object perception: Structure and process. Hillsdale, NJ: Erlbaum.

Shulman, G., Sullivan, M., Gish, K., \& Sakoda, W. (1986). The role of spatial-frequency channels in the perception of local and global structure. Perception, 15, 259-273.

Shulman, G., \& Wilson, J. (1987). Spatial frequency and selective attention to local and global information. Perception, 16, 89-101.

SINGER, W., \& BEDWORTH, N. (1973). Inhibitory interactions between $\mathrm{X}$ and $\mathrm{Y}$ units in the cat's lateral geniculate nucleus. Brain Research, 49, 291-307.

Tolmurst, D. J. (1975). Reaction times in the detection of gratings by human observers: A probabilistic mechanism. Vision Research, $15,1143-1149$.

VAN KLEECK, M. H. (1989). Hemispheric differences in global versus local processing of hierarchical visual stimuli by normal subjects: New data and a meta-analysis of previous data. Neuropsychologia, 27, 1165-1178

(Manuscript received February 7, 1991; revision accepted for publication May 24, 1993.) 